\title{
Therapeutic Effects of Genetically Modified Wharton's Jelly Mesenchymal Stem Cells Expressing Erythropoietin on Cancer-related Anemia in Mice Model
}

\section{Mohammad Estiri}

Spaceage Medicine BV, Rotterdom, Netherlands

\section{Bahareh Estiri}

Space Medicine BV, Rotterdom, Netherlands

\section{Asghar Fallah}

Iranian Institute of Cell \& Gene therapy, Tehran, Iran

\section{Marzyeh Aghazadeh}

Stem Cell Research Center, Tabriz University of Medical Sciences

\section{Amir Sedaqati}

Space Medicine BV

\section{Abbas Abdollahi}

Department Of Medical Microbiology, Fasa University of Medical Sciences, Iran

\section{Mahsa Rabienia}

Fasa University of Medical Science

\section{Nahid Mortazavidehkordi}

Department of Medical Parasitology, Fasa University of Medical Sciences, Fasa, Iran

Akbar Farjadfar ( $\nabla$ farjadbio@gmail.com )

Department of Medical Biotechnology, Fasa University of Medical Sciences, Fasa, Iran.

\section{Research}

Keywords: Cell and gene therapy, Cancer-related anemia, Erythropoietin, Lentivirus, Mesenchymal stem cells, Mice model of cancer

Posted Date: October 2nd, 2020

DOl: https://doi.org/10.21203/rs.3.rs-84099/v1

License: (c) (i) This work is licensed under a Creative Commons Attribution 4.0 International License. Read Full License 


\section{Abstract}

\section{Background}

Cancer-related anemia (CRA) negatively influences cancer patients' survival, disease progression, treatment efficacy, and quality of life (QOL). Current treatments such as iron therapy, red cell transfusion, and erythropoietin-stimulating agents (ESAs) may cause severe adverse effects. Therefore, development of long-lasting and curative therapies is highly required. Combined cell and gene therapy platform can introduce a new route for permanent production of erythropoietin (EPO) in the body with various degrees of clinical benefits and avoiding the need for repeat treatments.

\section{Methods}

In this study, we developed cell and gene therapy strategy for in-vivo delivery of EPO cDNA via genetic engineering human Wharton's jelly mesenchymal stem cells (hWJMSCs) to long-term produce and secret human EPO protein after transplantation into the mice model of CRA. To evaluate CRA's treatment in cancer-free and cancerous conditions, at first, we designed recombinant breast cancer cell line 4T1 expressing herpes simplex virus type 1 thymidine kinase (HSV1-TK) by a lentiviral vector encoding HSV1TK and injected into mice. After 3 weeks, all mice develop the metastatic breast cancer associated to the acute anemia. Then, we administrated ganciclovir (GCV) for 10 days in half of the mice to clear cancer cells. Meanwhile, we designed another lentiviral vector encoding EPO to transduce hWJMSCs. Following implantation of rhWJMSCs-EPO, the whole peripheral blood samples were collected from the tail vein once per week which were immediately analyzed for the measurements of EPO, hemoglobin $(\mathrm{Hb})$, and hematocrit (Hct) plasma levels.

\section{Results}

We found that after implantation of rhWJMSCs-EPO, plasma EPO, $\mathrm{Hb}$, and Hct concentration significantly increased which rose to a peak in the fourth week and remained at a therapeutic level for $>17$ weeks in the cancer-free group and $>10$ weeks in the cancerous group.

\section{Conclusion}

Our data indicate that the EPO-transduced hWJMSCs could improve the anemia of cancer in both cancerfree and cancerous mice model. This significant difference in length of time that $\mathrm{Hb}$ and $\mathrm{Hct}$ are in therapeutic levels in both treatment groups indicates that developing a precise targeted-therapy to eliminate cancer cells along with an effective treatment for CRA, as we presented here, could bring important clinical benefits.

\section{Background}

Cancer-related anemia (CRA), as a common consequence of tumor burden, occurs in more than $30 \%$ of cancer patients at diagnosis time which can reach $90 \%$, when the patients go under aggressive chemo- 
radiotherapy $(1,2)$. Global demographic characteristics predict an increased cancer incidence in the next decades which will impose a considerable challenge to healthcare systems(3). The mechanisms contributed to CRA are included into chemotherapy-induced anemia (CIA), blood loss, iron deficiency, erythropoietin deficiency due to renal disease, and bone marrow involvement with the tumor(4). Also, cancer cells alter the production of several inflammatory cytokines including tumor necrosis factor-alpha (TNFa), interleukin-6 (IL6), IL-1, interferon (IFN), and hepcidin which lead to less production of red blood cells (RBC) by suppressing the erythroid progenitor and also negative impact on erythropoietin via infiltrating into the bone marrow such as kidney $(5,6)$. Due to CRA's negative effect on survival, disease progression, treatment efficacy, and the patient's quality of life $(\mathrm{QOL})$, developing effective therapeutics for CRA is in great demand.

Current treatments for CRA include iron therapy, red cell transfusion, and erythropoietin-stimulating agents $(E S A s)(7,8)$. Erythropoietin (EPO) is a $30.4 \mathrm{kDa}$ glycoprotein hormone primarily produced by the fetal liver and adult kidney, which plays an important role in the body such as erythropoiesis, tissueprotective effects, and immune regulatory effects on immune cells(9). The administration of recombinant human erythropoietin (rhEPO) as a key regulator in the production of functional red blood cells is approved to treat the anemia of patients with long-lasting diseases such as cancer and renal failure with clinical benefits in correcting hemoglobin levels and markedly reduced the required number of blood transfusion and so, patients benefit from advantages, such as improved cardiac function, enhanced exercise capacity, and better QOL $(10,11)$. However, rhEPO administration may cause some adverse effects, including the possibility of host immunity against rhEPO, frequent self-administration by the patients which may not know the correct injection method and high cost(12).

Gene therapy via designing the plasmid DNA and viral vectors encoding the EPO gene introduced an attractive research area for treating anemia. In preclinical studies, gene therapy for direct delivery of EPO gene into animal models' skeletal muscle has shown a significant increase in EPO and erythropoiesis; however, life-threatening polycythemia and host immune responses to viral vectors limit its utilization in the clinic $(13,14)$. Cell-based therapies were studied as a treatment option in animal and clinical phases since 1990, which showed the satisfactory safety and efficacy profiles(15-17). Furthermore, the combined cell and gene therapy platform which we propose here is emerging as a potential alternative treatment to the traditional pharmacologic and also direct gene therapy; since it can permanently produce therapeutic proteins in the body with various degrees of clinical benefits and avoiding the need for repeat treatments(18-21). An effective cell and gene therapy protocol approach to deliver the EPO have more clinical and economic benefit than the repeated injection of EPO protein. Mesenchymal stem cells (MSCs) as desirable cell carriers can be easily obtained, expanded, and genetically engineered to express and secret therapeutic proteins in-vivo(21-24). Human Wharton's jelly mesenchymal stem cells (hWJMSCs) are multipotent stem cells which showed the potential to differentiate into mesodermal, ectodermal and endodermal lineages $(25,26)$. Compared to adult and fetal stem cells, hWJMSCs show a higher proliferation rate and minimum stimulation of immune and inflammatory systems(27-29). So, they have newly emerged as an appropriate therapeutic vehicle for gene therapy and drug delivery. Their therapeutic 
applications in various disease models, including inflammatory and autoimmune diseases, and cancer are being studied(30-32).

This investigation genetically modified hWJMSCs to long-term produce and secret human EPO protein after transplantation into CRA mice. To evaluate CRA's treatment in cancer-free and cancerous conditions, we genetically altered breast cancer cell line $4 \mathrm{~T} 1$ to express HSV-TK and inject into the mice. After confirming anemia induced by breast cancer, we eliminate cancer cells by administrating GCV in half of the mice. Following implantation of rhWJMSCs-EPO, plasma EPO, hemoglobin ( $\mathrm{Hb}$ ), and hematocrit (Hct) concentration significantly increased which indicate that the EPO-transduced hWJMSCs could improve the anemia of cancer in both cancer-free and cancerous mice model and can provide supporting evidence for future studies as a valuable therapeutic tool for the treatment of anemia.

\section{Materials And Methods Cell culture}

Human Embryonic Kidney 293T (HEK293T) and breast cancer cell line 4T1 were purchased from Mede Bioeconomy Company, Iran, and cultured in Dulbecco's modified Eagle's medium (DMEM; Gibco, Darmstadt, Germany). The cells were supplemented with 10\% fetal bovine serum (FBS) (Gibco, USA), penicillin (100 units/ml)/streptomycin $(100 \mathrm{mg} / \mathrm{ml})$ (Invitrogen, Carlsbad, CA, USA). Finally, the cells were incubated in a humidified atmosphere with $5 \% \mathrm{CO} 2$ at $37^{\circ} \mathrm{C}$.

\section{Gene design}

The coding sequences (CDS) of EPO (Accession\#: XM_001468996.1) were retrieved from GeneBank, NCBI. The sequences were synthesized by Genscript, USA, and were incorporated into pUC57 plasmid. In this experimental study, dual promoter lentiviral vector, $\mathrm{pCDH}-513 \mathrm{~B}$, was purchased from System Bio, USA. The first promoter is the cytomegalovirus (CMV) promoter with a downstream multiple cloning site (MCS) used for gene cloning. The second promoter is the EF1a promoter which regulates the expression of CopA-GFP (copepod green fluorescent protein) (cGFP) and puromycin resistance genes.

The Plasmid LOX-GFP-IRES-TK was obtained from Mede Bioeconomy Company, Iran. Then, EPO and herpes simplex virus type 1 thymidine kinase (HSV1-TK) fragments were sub-cloned into pCDH-513B under a CMV promoter with Spel-EcoR/ restriction enzymes (NEB, USA). To confirm the cloning procedure, the recombinant $\mathrm{pCDH}$-EPO and $\mathrm{PCDH}$-TK were digested using Xbal-Apal and Spel-EcoRI, respectively, and then sequenced.

\section{hWJMSCs isolation and characterization}

Human healthy umbilical cord (UCs) Wharton's jelly tissue was collected from full-term newborn and processed after obtaining the mother's informed consent. It was washed in phosphate-buffered saline (PBS) to eliminate the blood clots, disinfected, and cut into 1-2 mm lengths, and then were expanded on culture dishes with a minimum of Dulbecco's Modified Eagle Medium containing Nutrient Mixture F-12 
(DMEM/F12) medium (Gibco, USA), supplemented with 30-40\% FBS. Then, it was incubated at $37^{\circ} \mathrm{C}$ with $5 \% \mathrm{CO} 2$ which routinely monitored. After one week, solid umbilical cord pieces were removed, and cell migration was evaluated under the invert light microscope. Upon reaching $90 \%$ cell confluence, in the second changing medium, the adherent cells detached by the addition of $0.25 \%$ Trypsin-Ethylene

Diamine. For adipogenic and osteogenic differentiation, a six-well plate was cultured with $1 \times 10^{5}$ cells per well. After reaching a $40-45 \%$ cell confluence, an adipogenic differentiation medium (Gibco, USA) and osteogenesis supplement (Gibco, USA) were added to the basic medium, respectively. After about 20 days, lipid droplets were visualized using Oil Red O (Sigma-Aldrich, USA) staining. Successful osteogenic differentiation was verified by Alizarin Red (Sigma-Aldrich, USA staining. In the control group, hWJMSCs were grown in the culture medium without adipogenic and osteogenesis supplement. A small number of undifferentiated hWJMSCs (passage $3,10^{5}$ cells) were analyzed using BD FACSCalibur flow cytometry (BD Bioscience, USA) to evaluate surface markers expressed by hWJMSCs. After adding specific antibodies at the recommended concentrations, the tubes were incubated in the dark at room temperature for 30-60 minutes. Then, flow cytometry analysis was performed, and data were analyzed using FlowJo (version 7.6.1) software.

\section{Lentivirus production}

HEK293T was used to produce two recombinant lentivirus rLV-GFP, rLV-EPO, and rLV-TK using transfer vectors $\mathrm{pCDH}, \mathrm{pCDH}-\mathrm{EPO}$, and $\mathrm{pCDH}-\mathrm{TK}$, respectively. To do this, due to Prof. Trono lab protocol, CaPO4 transfection of HEK293T cells was performed with some modifications using the following amounts of DNA: $21 \mu \mathrm{g}$ transfer/control vector, $10.5 \mu \mathrm{g}$ pMD2.G vector, $15 \mu \mathrm{g}$ pMDLg/pRRE vector, and $13 \mu \mathrm{g}$ pRSVRev vector which all were dissolved in HEPES buffered water to reach $921 \mu \mathrm{l}$. Then, $33 \mu \mathrm{l}$ Tris-EDTA (TE) buffer was added, and the mixture was strongly mixed and kept at room temperature for 3 minutes. Then, $105 \mu \mathrm{l} \mathrm{CaCl} 22.5 \mathrm{M}$ was added, and the mixture was strongly vortexed and left for 3 min for making DNA$\mathrm{CaCl} 2$ interaction. Then, $1050 \mu \mathrm{l} \mathrm{HEPES} 2 \mathrm{X}$ was added while the mixture was being vortexed. HEK293T cells $\left(2 \times 10^{6}\right.$ cells $)$ medium was changed $2 \mathrm{~h}$ before transfection. HEK293T cells with $80 \%$ confluency were co-transfected by plasmids in a way that $2100 \mu \mathrm{l}$ transfection master mix was added per $10 \mathrm{~cm}$ HEK293T cells as a droplet to all areas of the plate. Then, they were incubated at $37^{\circ} \mathrm{C}$ in $5 \% \mathrm{CO} 2 \mathrm{for}$ $16 \mathrm{~h}$.

After 24 hours, the transfection efficiency was assessed by GFP expression and visualized by an inverted fluorescent microscope (Leica, German). We selected five fields randomly under a fluorescent microscope, and the percentage of GFP-positive cell numbers determined the transfection efficiency to total cell number. Mediums containing the virus were collected after 24,48 , and $72 \mathrm{~h}$ following transfection which were passed through $0.25 \mu \mathrm{m}$ pore filters to remove cellular debris. Recombinant lentivirus concentration was measured by the addition of polyethylene glycol (PEG) $60050 \%, \mathrm{NaCl} 4 \mathrm{M}$, and PBS to recombinant viruses inside polypropylene bottles which stored at $4{ }^{\circ} \mathrm{C}$ for 1.5 hours. Then, the tubes were centrifuged at $15000 \mathrm{rpm}$ for 15 minutes at $4{ }^{\circ} \mathrm{C}$. Fresh recombinant titrated viruses at the volumes of $1000,500,100$, 50, 20 and $0 \mu$ l were used for transducing hWJMSCs and $4 \mathrm{~T} 1$ cells.

\section{Transduction and viability assay}


The second passage of hWJMSCs and 4T1 cells were cultured at low confluency of $30-40 \%$ in a 6-well dish and incubated at $37^{\circ} \mathrm{C}, 5 \% \mathrm{CO} 2$ overnight. Then, they were transduced by rLV-GFP, rLV-EPO, and rLVTK to generate rhWJMSCs, rhWJMSCs-EPO, and r4T1-TK respectively. Cell transduction was evaluated using a fluorescent microscope, 72 hours after the transduction, and was compared with non-transduced MSCs and 4T1. Puromycin $(1.5 \mu \mathrm{g} / \mathrm{ml})$ selection started 72 hours after the transduction for the next 5 days.

For MTT assay, we cultured $5 \times 10^{3}$ cells from transduced and normal hWJMCSs and 4T1 cells in 96wells plates. After 1 day, we added MTT reagents and incubated for 4 hours. Also, to measure r4T1-TK cells' sensitivity to GCV, we add $20 \mu \mathrm{g} / \mathrm{ml} \mathrm{GCV}$ to the r4T1-TK cells culture medium. Then, we added the dimethyl sulfoxide (DMSO) to terminate the reaction and the plate was read at $570 \mathrm{~nm}$ wavelength using BioTek Instruments (Vermont, United States) microplate reader.

\section{Western blot analysis}

EPO and TK gene expression was confirmed after the transduction by western blotting assay. rhWJMSCsEPO and r4T1-TK supernatants were collected 72 hours after the transduction. Evaluating the protein concentrations was examined using a BCA Protein Assay Kit (Thermo Fisher, USA). Equivalent amounts of proteins ( $30 \mu \mathrm{g} /$ lane) were loaded onto $12 \%$ sodium dodecyl sulfate-polyacrylamide gel electrophoresis (SDS-PAGE) and then transferred onto nitrocellulose membranes (Bio-Rad, USA). The membranes were blocked using $5 \%$ non-fat milk, and immunoblotting was performed using antibodies against EPO and TK (Santa Cruz, USA). Proteins of interest were detected using HRP-conjugated sheep anti-mouse IgG antibody (Abcam, ab6785). Finally, the protein bands were visualized using chemiluminescence $(E C L)$ reagent, and the integrated optical density (IOD) of each protein band was measured. The internal standard $\beta$-actin adjusted IOD values.

\section{CRA mice model}

The mice were obtained from the laboratory animal center of Pasteur Institute of Iran. Male and female BALB/c mice (6- to 8 weeks old, $n=60$; weight, $18-20 \mathrm{~g} ; \mathrm{n}=10 \mathrm{mice} / \mathrm{group}$ ) were housed and treated in a pathogen-free environment with the access to autoclaved food and water ad libitum according to national rules on animal experiments. Also, the mice were maintained on an iron-sufficient diet for 2 weeks before the injection of tumor cells or saline, which continued until the end of the study(33). $5 \times 10^{5}$ recombinant 4T1-TK cells diluted in $100 \mu \mathrm{l}$ PBS were injected into the right fourth mammary gland of mice using a 25-gauge needle(34). The tumor was palpably detected after one week of injection in all 60 mice; thereafter, twice a week routinely, the tumor volume was measured until the end of the study. Because r4T1-TK tumors were metastatic within 2-3 weeks after injection, to evaluate the consequences of tumor progression on erythropoiesis compare to the control group, peripheral blood samples were obtained from the tail vein at week 3 and were analyzed using a Sysmex XT-2000i automated hematology analyzer (Sysmex Corp., Hyogo, Japan) for the measurements of red blood cell (RBC) count, reticulocyte numbers, $\mathrm{Hc}$, and $\mathrm{Hb}$ concentration to confirm anemia in mice. Those r4T1-TK-bearing BALB/c mice qualified for developing the anemia were randomly divided into 6 groups $(n=10$ 
mice/group). In this study, taking into account our published findings in cancer-free and cancerous mice, three groups of animals were treated intraperitoneal (IP) injection with Ganciclovir (GCV, $100 \mathrm{~mL}$ ) at $75 \mathrm{mg} / \mathrm{kg}$ twice daily for 10 continuous days(35). The other 3 groups of animals received PBS for the same period. The experimental animal protocols were performed due to the Guide for the Care and Use of Laboratory Animals published by the US National Institutes of Health.

\section{Implantation of rhWJMSCs-EPO}

We evaluated our anemia treatment protocol in six groups of animals, all of which had the anemia (3 cancer-free groups and 3 cancerous groups) according to the following instructions: A: cancer-free mice that received a moderate dose of rhWJMSCs-EPO to evaluate the effects of treatment during cancer suppression. B: control cancer-free mice that received control rhWJMSCs. C: control cancer-free mice that received PBS. D: control cancerous mice that received rhWJMSCs-EPO to evaluate treatment effects during the active phase of cancer. E: cancerous control mice which received rhWJMSCs. F: control cancerous mice which received PBS. To avoid polycythemia, we used a moderate dose of rhWJMSCsEPO $\left(\sim 7 \times 10^{6}\right)$ implanted into mice's skeletal muscle with a $29-\mathrm{G}$ insulin syringe because, according to some studies, the high dose of treatment $\left(10 \times 10^{6}\right)$ can develop the polycythemia(36).

For laboratory measurements, the whole peripheral blood samples were collected from the tail vein once per week which were immediately analyzed for the measurements of Hct and $\mathrm{Hb}$ plasma levels. Moreover, the changes in plasma EPO levels in all CRA mice were detected by ELISA (Quantikine ELISA kit, R\&D Systems, Minneapolis, MN, USA) according to the manufacturer's protocol.

\section{Statistics}

All data are present as mean \pm standard error of the mean (S.E.M.). The statistical analysis of data was performed using the Student's t-test and analyzed by Prism software (GraphPad, San Diego, CA). P-value $<0.05$ were considered statistically significant.

\section{Results}

\section{Lentiviral vectors construction}

Vectors containing EPO and HSV-TK were constructed using the DNA assembling method. Human EPO and HSV-TK cDNA were cloned in PCDH-513B-1 lentiviral vector (Fig. 1A, C). Both genes were confirmed following enzymatic digestion and sequencing. The digestion of $\mathrm{pCDH}-\mathrm{EPO}$ using $\mathrm{Xbal}$ and Apel produced two bands of 1900 and 7800 base pair (bp) (Fig. 1B), and digestion of the HSV-TK gene with Spel and EcoR/ showed two bands of 1100 and 8200 bp (Fig. 1D). Sequencing was done for final confirmation. In this construct, EPO and HSV-TK mRNA were transcripts from CMV promoter, and cGFP and Puromycin mRNA were transcriptions from the EF1a promoter.

\section{hWJMSCs isolation and characterization}


The hWJMSCs derived from human UC were isolated and cultured based on cell migration and surface attachment. In the third passage, the identity and properties of isolated hWJMSCs were verified by immunophenotyping of cell-surface antigens. According to flow cytometry analysis hWJMSCs were highly positive for CD105 and CD90 surface markers and negative for hematopoietic markers, CD 45 and CD34 (Fig. 2A). The fibroblastic-like morphology of the cells was confirmed under the inverted microscope. The hWJMSCs had a typical spindle shape with a capacity to differentiating into osteogenic and adipogenic lineages (Fig. 2B). The accumulation of lipid vacuoles was evaluated by Oil Red staining, and Alizarin Red revealed calcium deposition.

\section{Lentivirus production, In-vitro transduction and viability assay}

GFP reporter gene in the lentiviral vector was an index for the transfection and transduction efficiency. Transfer and control vectors were co-transfected in HEK293T cells with the helper packaging vectors based on CaPO4 protocol. The transfection efficiency due to the GFP positive and negative cells counted under a fluorescent microscope was higher than 90\% (Fig. 3A). Recombinant virus titrations were done using quantitative polymerase chain reaction (qPCR), and fresh viral particle titration was approximately 1.5-2 $\times 10^{6}$ particles. We transduced 4T1 cells with rLV-TK and hWJMSCs with rLV-GFP and rLV-EPO and then cultured in a medium containing puromycin to separate them from non-transduced cells. The fluorescent microscope visualized the expression rate of GFP. The transduction of hWJMSCs and 4T1 cells with concentrated and fresh recombinant viruses does not show any significant difference (Fig. 3B).

MTT assay showed that transduction and genome integration of transfer lentiviral vectors do not significantly affect the viability of both transduced hWJMSCs and 4T1 compared with non-transduced cells (Fig. 3C). As TK activates by GCV, we tested the sensitivity of r4T1-TK cells to GCV by adding $20 \mu \mathrm{g} / \mathrm{ml} \mathrm{GCV}$ to the r4T1-TK cells culture medium. Results indicated that r4T1-TK cells' survival decreased with the addition of GCV drug (Fig. 3C).

Western blot results confirmed gene expression data at the protein level. Western blot analysis showed similar expression levels for $\beta$-actin protein in control and transduced hWJMSCs and 4T1 cells, compared to EPO and TK protein which was only overexpressed in transduced hWJMSCs and 4T1 cells, respectively (Fig. 3D). These results showed that both proteins (EPO and TK) were transcribed and translated correctly.

\section{CRA mice model}

Generating mice model of CRA, the recombinant r4T1-TK was injected into mice to develop breast cancer and anemia as a result of cancer development. At 14 days after injection, all tumor mice appeared in distress, as evidenced by lethargy and poor feeding secondary to tumor load. At first, the consequence of tumor burden on erythropoiesis was evaluated via analyzing RBC counts, $\mathrm{Hb}$ concentrations, $\mathrm{Hct}$, and reticulocyte counts in the peripheral blood samples of r4T1-TK tumor-bearing mice at week 3. r4T1-TKbearing mice showed an anemic feature with lower RBC count, $\mathrm{Hb}$, and Hct levels, as well as increased 
reticulocytosis, compared to control mice (Fig. 4A). After confirming the presence of anemia in all tumor mice, we treated three groups with GCV drug, whereas the other three groups received PBS, as mentioned in Material and Methods. As expected, we observed a tumor suppression in mice which received a $75 \mathrm{mg} / \mathrm{kg}$ dose of GCV drug than the PBS groups in which tumor volume consistently increased (Fig. 4B).

\section{Effects of treatment}

Our experimental procedures to evaluate the time course of the biological effect of EPO-secreting rhWJMSCs on the anemia of cancerous and cancer-free mice with experimental breast cancer is described in Materials and Methods which illustrated in Fig. 4. Among cancer-free groups (A, B, and C), plasma EPO level in group $\mathbf{A}$ which received rhWJMSCs-EPO, significantly raised and reached number $105.4 \pm 23 \mathrm{mU} / \mathrm{ml}$ at week 4, compared to its two control groups $\mathbf{B}$ and $\mathbf{C} \sim 15 \pm 2.8 \mathrm{mU} / \mathrm{ml}$ which received rhWJMSCs and PBS, respectively (Fig. 5A). This model of change detected in cancerous groups (D, E, and F) in which plasma EPO level was $103.1 \pm 21.6 \mathrm{mU} / \mathrm{ml}$ in group $\mathrm{D}$, which received rhWJMSCs-EPO in compare to its two control groups $E$ and $F \sim 17.5 \pm 3.2 \mathrm{mU} / \mathrm{ml}$ which received rhWJMSCs and PBS, respectively (Fig. 5A). Although there was a significant difference in EPO level between each treatment group ( $A$ and $\mathbf{D}$ ) and its control groups, we found a slight difference in EPO level between two treatment groups A and B.

After 7 days of intramuscular-implanted rhWJMSCs-EPO, an increase in $\mathrm{Hb}$ was achieved in A and D groups. However, compared to control groups which receive rhWJMSCs and PBS, they showed little difference in the first week after the treatment (Fig. 5B). The increase in hemoglobin becomes more substantial in two treatment groups per week 4 in which in group $A$, hemoglobin level rose to level $17.2 \pm$ $2.3 \mathrm{~g} / \mathrm{dl}$, to compare with group D $15.5 \pm 1.8 \mathrm{~g} / \mathrm{dl}$. So, although hemoglobin level rose to its highest number in group $\mathbf{D}$, it did not increase as much as the amount of hemoglobin in group $\mathbf{A}$. Thus, we noticed a considerable difference in response to EPO treatment in cancer-free and cancerous groups. This different response to treatment was more noticeable after observing similar hemoglobin changes pattern among control groups ( $B$ and $\mathbf{C}$ vs. $\mathbf{E}$ and $\mathbf{F}$ ) which didn't receive rhWJMSCs-EPO in which cancer-free groups $\mathbf{B}$ and $\mathbf{C}$ showed more increase in hemoglobin compare with cancerous groups $E$ and $F$ (Fig. 5B). It was present, while we had a similar pattern of changes in the rate of Hct increase in response to treatment. The Hct in group $A$, as in group $D$, reached the therapeutic level, more precise, to $60.3 \% \pm 2.7$ in group $\mathbf{A}$ and $51.5 \% \pm 1.4$ in group $\mathrm{D}$, by approximately 4 weeks after rhWJMSCs-EPO transplantation, whereas the Hct was not significantly altered in control groups mice which received the control rhWJMSCs and PBS (Fig. 5C). Although the amount of Hct in cancer-free control groups (B and C) increased further compare to cancerous control groups ( $E$ and $F$ ), none of them reached therapeutic levels, similar to what was seen in $\mathrm{Hb}$ level of control groups (Fig. 5C).

In general, we found a significant correlation between the amount of plasma level of EPO and $\mathrm{Hb}$ and also Hct concentration. In both groups of $A$ and $D$ which received $\sim 7 \times 10^{6}$ rhWJMSCs-EPO, the $\mathrm{Hb}$ and Hct which had declined from basal $16.1 \pm 2.1$ to $9.3 \pm 0.7$ and $51 \% \pm 1.2$ to $31.7 \% \pm 0.5$ respectively, approximately 3 weeks after r4T1-TK injection, reach the therapeutic level at week 4 . As we measured the 
plasma level of EPO weekly, we found a decrease in its level after week 5, but stayed in therapeutic level for $>17$ weeks in both groups A and D. As EPO decreased, the plasma level of Hb and Hct decline in both treatment groups. However, $\mathrm{Hb}$ and $\mathrm{Hct}$ concentration persist at a therapeutic level for $>17$ weeks for group $\mathbf{A}$ and for $>10$ weeks for group $\mathbf{D}$.

\section{Discussion}

Our study on CRA treatment has included cell and gene therapy approaches, whereby the EPO gene is transferred to hWJMSCs by lentiviral vectors in-vitro, and the cells are subsequently implanted in-vivo to serve as EPO-releasing vehicles to establish, if EPO significantly increased $\mathrm{Hb}$ and hct levels.

Several studies have reported using gene therapy to deliver the EPO gene using plasmid DNA and viral vectors such as adenoviral and adeno-associated viral (AAV) vectors. By designing plasmid DNA expressing rat EPO gene and direct delivery into skeletal muscle of rat anemia model by subtotal nephrectomy, studies showed a noticeable increase in $\operatorname{Hct}(37,38)$. The administration of adenoviral or AAV vectors into an animal model of anemia to deliver EPO resulted in an increase in plasma level of EPO and erythropoiesis which introduce viral vectors as a highly efficient gene delivery vehicle(39, 40); however, life-threatening polycythemia was reported $(14,41)$. The erythropoiesis response to therapy was proportional to the dose of plasmid DNA or viral vectors delivered. Moreover, host immune responses to these vectors and their transgene products are associated to potential health risks limiting their entry into the clinical phase $(13,42)$.

One remedy to overcome the safety risks and the limitation of gene therapy approaches is using cells as delivery vehicles for plasma-soluble therapeutic proteins in-vivo like EPO, which allow us to quantify and control the serum level of EPO expressed by transduced cells through adjusting the number of implanted gene-modified cells secreting EPO to prevent severe polycythemia and also reduce the risk of systemic virus dissemination(43). MSCs are promising candidates for gene delivery to treat the hematological diseases like anemia, mostly due to their accessibility for genetic modification and the simplicity of their culture and expansion in vitro $(22,44)$. Indeed, some experimental studies were reported using the MSCs as a suitable delivery vehicle for therapeutic proteins in vivo $(36,45-47)$. Viral methods were widely used in the production of therapeutic protein by MSCs(48). The main purpose of our investigation was to apply this biopharmaceutical approach for the EPO delivery in vivo for the treatment of CRA. In this study, we isolated MSCs from the human UCs as a good source of MSCs, because they can be harvested noninvasively in large numbers after birth with no ethical problems compared to MSCs derived from adults, have some advantages such as an improved proliferative capacity, life span, differentiation potential, and immunomodulatory properties which offer the best clinical utility $(29,32,49)$.

We transduced hWJMSCs with an EPO-encoding lentiviral vector under highly controlled the conditions in vitro to avoid any risk of viral dissemination in vivo. Transplantation of a moderate dose of rhWJMSCsEPO $(\sim 7 \times 106)$ into the CRA mice model's skeletal muscle resulted in a cell dose-dependent increase of EPO level that reached up to $100 \mathrm{mU} / \mathrm{ml}$ in both treatment groups (A and $\mathbf{D}$ ) after 4 weeks. It remained 
high until the end of the study (>17 weeks) (Fig. 5A). Both $\mathrm{Hb}$ and Hct increase in response to EPO in both groups $\mathbf{A}$ and $\mathbf{D}$; however, the increase in $\mathrm{Hb}$ and $\mathrm{Hct}$ in cancer-free group $\mathbf{A}$ was more significant than the cancerous group D (Fig. 5B, C). Also, cancer-free control groups (B and C), in comparison to cancerous control groups ( $E$ and $F$ ), which received control treatments (rhWJMSCs and PBS), showed a higher level of $\mathrm{Hb}$ and $\mathrm{Hct}$. Whereas all control groups had a low level of EPO. Thus, we concluded that combined cell and gene therapy strategies for correcting CRA could be more effective if the cancer is treated at the same time. It is currently believed that chemoradiotherapy is the key means for treating cancer patients, making CRA worse, and other serious side effects $(2,50)$. So, developing advanced therapeutic procedures which precisely target cancer cells are in great demand. In this study, we engineered a recombinant 4T1 cells expressing HSV-TK to inject and develop breast cancer-associated anemia in mice, followed by injecting GCV to clear almost all cancer cells expressing TK in three groups of anemic animals. As a result, showed (Fig. 4B), three groups which receive GCV displayed a significant tumor regression compared to cancerous groups. So, we could evaluate the efficacy of rhWJMSCs-EPO in cancer-free and cancerous groups to correct CRA in which the cancer-free groups had no serious side effects or other organ damage due to cancer treatment by GCV. Although this cancer treatment has no clinical utilization and we just design it in our study to evaluate the effect of rhWJMSCs-EPO on CRA in the condition in which cancer is treated via a precise targeted-therapy method without serious side effects which we see in other methods like chemo-radiotherapy, this hypothesis has important clinical implications, because developing therapeutic methods that only target cancer cells and clear all of them without influencing other tissues or organs, similar to what we did as an animal study, not only can improve CRA over time, but also can pave the other CRA treatment such as cell and gene therapy that we used in this study.

We observed a gradual decrease in plasma concentration of $\mathrm{Hb}$ and Hct during $\sim 10$ weeks which continued for $>17$ weeks, in correlation to a decrease of EPO. It could be because, according to some studies, MSCs do not persist in the recipient organism for the prolonged periods $(51,52)$. We hypothesized a second dose of rhWJMSCs-EPO transplantation could be associated with satisfactory therapeutic results that need further investigation in MSC engineering and therapy. Consequently, we will able to schedule treatment plans in which MSCs transplantation courses will be done with determined doses depending on the disease stage. Therefore, the cell and gene therapy approach used here in our study have its limitations as a long-term approach to CRA therapy.

Supraphysiologic response leading to polycythemia may develop after the first transplantation of EPOsecreting hWJMSCs which may require resection of cells or, conversely as we mentioned earlier the modified MSCs lose their effectiveness over time and therefore, re-implantation may be required to enhance their clinical usage.

A clinical trial applying cell and gene therapy was performed in patients with anemia associated to chronic renal failure (CRF). In this study, the autologous dermal sample was transduced by an adenoviral vector expressing human EPO and transplanted subcutaneously into patients leading to a significant rise in plasma level of hEPO and an increase in reticulocyte count(53). The patient didn't develop serious side 
effects related to the treatment. However, the significant increase in $\mathrm{Hb}$ in patients didn't achieve which could be due to a short-time increase in hEPO. The transient increase in EPO concentration for approximately 10 days stems from the fact that the cellular immune response can develop against viral proteins expressed by the adenovirus(53). Besides, terminally differentiated fibroblasts which were used as cell vehicle has some limitations such as the non-sustained release of the desired secretory protein due to inactivation of vector sequence following transplantation, and also depend on donor's age the expansion capability of normal fibroblasts may be restricted because they ultimately reach a stage when the cell division cycle slow down leading to cell aging which limits their clinical applications(54). In contrast, hWJMSCs used in this study are attractive candidates due to their potential expansion ability, an immuno-privileged status, and easy access for collection, which afford us high-efficiency lentiviral engineering cells, culture, and utilization in vivo of selected modified cells(55-57).

\section{Conclusion}

Our data confirmed that administration of a moderate dose of rhWJMSCs carrying the EPO CDNA resulted in the elevated circulating level of EPO in the CRA mice model, which caused a persistent increase in $\mathrm{Hb}$ and $\mathrm{Htc}$. So, a combination of hWJMSCs and lentiviral vector could be suggested as a novel cell and gene therapy approach for CRA. When this anemia treatment protocol is combined with a precise targeted-therapy for cancer cells, it will give the best results. Obviously, there are challenges to the clinical use of cell and gene therapies for diseases such as anemia. We will watch closely for clinical safety and efficacy using these methods for disease treatment that is needed to keep this field ahead.

\section{Abbreviations}

AAV

Adeno-associated virus

CDS

Coding sequences

CIA

Chemotherapy-induced anemia

CMV

Cytomegalovirus

CRA

Cancer-related anemia

CRF

Chronic renal failure

DMEM

Dulbecco's modified Eagle's medium

ECL

Chemiluminescence 
EPO

Erythropoietin

ESAs

Erythropoietin-stimulating agents

FBS

Fetal bovine serum

GCV

Ganciclovir

$\mathrm{Hb}$

Hemoglobin

Hct

Hematocrit

HEK293T

Human embryonic kidney 293T

HSV1-TK

Herpes simplex virus type 1 thymidine kinase

hWJMSCs

Human wharton's jelly mesenchymal stem cells

IFN

Interferon

IL6

Interleukin-6

IOD

Integrated optical density

IP

Intraperitoneal

MCS

Multiple cloning site

MSCs

Mesenchymal stem cells

PBS

Phosphate-buffered saline

qPCR

Quantitative polymerase chain reaction

PEG

Polyethylene glycol

QOL

Quality of life

rhEPO

Recombinant human erythropoietin 


\section{S.E.M.}

Standard error of the mean

TE

Tris-EDTA

TNFa

Tumor necrosis factor-alpha

UC

Umbilical cord

\section{Declarations}

\section{Acknowledgements}

We thank Fasa University of Medical Sciences and all members from Department of Stem Cell Mede Bioeconomy, for their supports.

\section{Funding}

This work was supported by Fasa University of medical Sciences (Grant No. 97072)

Author information

\section{Affiliations}

Space Medicine BV, Rotterdam, Netherland

Mohammad Estiri, Bahareh Estiri, Amir Sedaqati

Iranian institute of Cell \& Gene Therapy (iiCGT), Tehran, Iran

Asghar Fallah

Stem Cell Research Center, Tabriz University of Medical Sciences, Tabriz, Iran

Marzyeh Aghazadeh

Department of Medical Microbiology, Fasa University of Medical Sciences, Fasa, Iran Abbas Abdollahi

Department of Medical Biotechnology, Fasa University of Medical Sciences, Fasa, Iran Mahsa Rabienia, Akbar Farjadfar

Department of Medical Parasitology, Fasa University of Medical Sciences, Fasa, Iran 


\section{Contributions M.E, B.E, A.F, M.A, A.S, A.A, A.F, M.R, N.M}

A.F., M.E, and B.E. contributed to experimental design, data interpretation, data analysis, and manuscript preparation. B.E and A.A made constructs and performed confirmation, and N.M subcloned constructs into $\mathrm{PCDH} 513 \mathrm{~B}$ for virus packaging. M.A and A.S isolated hWJMSC, Characterization of hWJMSC was performed by A.A and M.R under the supervision of A.F. Virus production was done by M.E, A.S, and M.A. M.E and M.R did in vivo study.

\section{Corresponding author}

Correspondence to Akbar Farjadfar.

\section{Ethics declarations}

\section{Ethics approval and consent to participate}

The experimental animal protocols were performed due to the Guide for the Care and Use of Laboratory Animals published by the US National Institutes of Health. This study was approved by Iranian National Committee for Ethics in Biomedical Research.

\section{Consent for publication}

Not applicable.

\section{Competing interests}

The authors have declared that no competing interest exists.

\section{References}

1. Gilreath JA, Stenehjem DD, Rodgers GM. Diagnosis and treatment of cancer-related anemia. Am J Hematol. 2014;89(2):203-12.

2. Madeddu C, Gramignano G, Astara G, Demontis R, Sanna E, Atzeni V, et al. Pathogenesis and treatment options of cancer related anemia: perspective for a targeted mechanism-based approach. Frontiers in physiology. 2018;9:1294.

3. Pilleron S, Sarfati D, Janssen-Heijnen M, Vignat J, Ferlay J, Bray F, et al. Global cancer incidence in older adults, 2012 and 2035: a population-based study. International journal of cancer. 2019;144(1):49-58.

4. Sankaran VG, Weiss MJ. Anemia: progress in molecular mechanisms and therapies. Nature medicine. 2015;21(3):221-30.

5. Dicato M, Plawny L, Diederich M. Anemia in cancer. Ann Oncol. 2010;21(suppl_7):vii167-72. 
6. Birgegård G, Aapro MS, Bokemeyer C, Dicato M, Drings $P$, Hornedo J, et al. Cancer-related anemia: pathogenesis, prevalence and treatment. Oncology. 2005;68(Suppl. 1):3-11.

7. Abdel-Razeq $\mathrm{H}$, Hashem $\mathrm{H}$. Recent update in the pathogenesis and treatment of chemotherapy and cancer induced anemia. Crit Rev Oncol/Hematol. 2020;145:102837.

8. Schrijvers D. Management of anemia in cancer patients: transfusions. Oncologist. 2011;16.

9. Peng B, Kong G, Yang C, Ming Y. Erythropoietin and its derivatives: from tissue protection to immune regulation. Cell Death Dis. 2020;11(2):1-12.

10. Debeljak N, Solár P, Sytkowski AJ. Erythropoietin and cancer: the unintended consequences of anemia correction. Frontiers in immunology. 2014;5:563.

11. Gouri Shankar A. The role of recombinant erythropoietin in childhood cancer. Oncologist [Internet]. 2008 [citado el 24 de abril 2009]; 13 (3):[aprox. 2 p.]..

12. Salamin $O$, Kuuranne T, Saugy M, Leuenberger $N$. Erythropoietin as a performance-enhancing drug: its mechanistic basis, detection, and potential adverse effects. Molecular cellular endocrinology. 2018;464:75-87.

13. Shirley JL, de Jong YP, Terhorst C, Herzog RW. Immune responses to viral gene therapy vectors. Mol Ther. 2020;28(3):709-22.

14. Johnston J, Tazelaar J, Rivera VM, Clackson T, Gao G-P, Wilson JM. Regulated expression of erythropoietin from an AAV vector safely improves the anemia of $\beta$-thalassemia in a mouse model. Mol Ther. 2003;7(4):493-7.

15. Wood EH, Tang PH, De la Huerta I, Korot E, Muscat S, Palanker DA, et al. Stem cell therapies, genebased therapies, optogenetics, and retinal prosthetics: current state and implications for the future. Retina (Philadelphia Pa). 2019;39(5):820.

16. Shomali N, Gharibi T, Vahedi G, Mohammed RN, Mohammadi H, Salimifard S, et al. Mesenchymal stem cells as carrier of the therapeutic agent in the gene therapy of blood disorders. Journal of cellular physiology. 2020;235(5):4120-34.

17. Gimble JM, Guilak F, Bunnell BA. Clinical and preclinical translation of cell-based therapies using adipose tissue-derived cells. Stem Cell Res Ther. 2010;1(2):19.

18. Dwyer RM, Khan S, Barry FP, O'Brien T, Kerin MJ. Advances in mesenchymal stem cell-mediated gene therapy for cancer. Stem Cell Res Ther. 2010;1(3):1-7.

19. Cho IK, Hunter CE, Ye S, Pongos AL, Chan AWS. Combination of stem cell and gene therapy ameliorates symptoms in Huntington's disease mice. NPJ Regenerative medicine. 2019;4(1):1-9.

20. Fierro FA, Magner N, Beegle J, Dahlenburg H, Logan White J, Zhou P, et al. Mesenchymal stem/stromal cells genetically engineered to produce vascular endothelial growth factor for revascularization in wound healing and ischemic conditions. Transfusion. 2019;59(S1):893-7.

21. Han J, Liu Y, Liu H, Li Y. Genetically modified mesenchymal stem cell therapy for acute respiratory distress syndrome. Stem Cell Res Ther. 2019;10(1):386. 
22. Wei W, Huang Y, Li D, Gou H-F, Wang W. Improved therapeutic potential of MSCs by genetic modification. Gene therapy. 2018;25(8):538-47.

23. Venkatesan JK, Ekici M, Madry H, Schmitt G, Kohn D, Cucchiarini M. SOX9 gene transfer via safe, stable, replication-defective recombinant adeno-associated virus vectors as a novel, powerful tool to enhance the chondrogenic potential of human mesenchymal stem cells. Stem Cell Res Ther. 2012;3(3):22.

24. Dhoke NR, Kaushik K, Das A. CXCR6-based mesenchymal stem cell gene therapy potentiates skin regeneration in murine diabetic wounds. Molecular Therapy. 2020.

25. Lo Furno D, Mannino G, Giuffrida R, Gili E, Vancheri C, Tarico MS, et al. Neural differentiation of human adipose-derived mesenchymal stem cells induced by glial cell conditioned media. J Cell Physiol. 2018;233(10):7091-100.

26. Shi Q, Gao J, Jiang Y, Sun B, Lu W, Su M, et al. Differentiation of human umbilical cord Wharton's jelly-derived mesenchymal stem cells into endometrial cells. Stem Cell Res Ther. 2017;8(1):1-12.

27. Sabapathy V, Sundaram B, Sreelakshmi V, Mankuzhy P, Kumar S. Human Wharton's jelly mesenchymal stem cells plasticity augments scar-free skin wound healing with hair growth. PloS one. 2014;9(4):e93726.

28. Kern S, Eichler H, Stoeve J, Klüter H, Bieback K. Comparative analysis of mesenchymal stem cells from bone marrow, umbilical cord blood, or adipose tissue. Stem cells. 2006;24(5):1294-301.

29. Millán-Rivero JE, Nadal-Nicolás FM, García-Bernal D, Sobrado-Calvo P, Blanquer M, Moraleda JM, et al. Human Wharton's jelly mesenchymal stem cells protect axotomized rat retinal ganglion cells via secretion of anti-inflammatory and neurotrophic factors. Scientific reports. 2018;8(1):1-14.

30. Hosseini A, Estiri H, Niaki HA, Alizadeh A, Zadeh BA, Ghaderian SMH, et al. Multiple Sclerosis Gene Therapy Using Recombinant Viral Vectors: Overexpression of IL-4, IL-10 and Leukemia Inhibitory Factor in Wharton's Jelly Stem Cells in The EAE Mice Model. Cell Journal (Yakhteh). 2017;19(3):361.

31. El Omar R, Beroud J, Stoltz J-F, Menu P, Velot E, Decot V. Umbilical cord mesenchymal stem cells: the new gold standard for mesenchymal stem cell-based therapies? Tissue Engineering Part B: Reviews. 2014;20(5):523-44.

32. Ye F, Jiang J, Zong C, Yang X, Gao L, Meng Y, et al. Sirt1-overexpressing Mesenchymal stem cells drive the anti-tumor effect through their pro-inflammatory capacity. Mol Ther. 2020;28(3):874-88.

33. Kim A, Rivera S, Shprung D, Limbrick D, Gabayan V, Nemeth E, et al. Mouse models of anemia of cancer. PLoS One. 2014;9(3):e93283.

34. Liu M, Jin X, He X, Pan L, Zhang X, Zhao Y. Macrophages support splenic erythropoiesis in 4T1 tumor-bearing mice. PLoS One. 2015;10(3):e0121921.

35. Majumdar AS, Zolotorev A, Samuel S, Tran K, Vertin B, Hall-Meier M, et al. Efficacy of herpes simplex virus thymidine kinase in combination with cytokine gene therapy in an experimental metastatic breast cancer model. Cancer Gene Ther. 2000;7(7):1086-99.

36. Eliopoulos N, Gagnon RF, Francois M, Galipeau J. Erythropoietin delivery by genetically engineered bone marrow stromal cells for correction of anemia in mice with chronic renal failure. J Am Soc 
Nephrol. 2006;17(6):1576-84.

37. Sebestyén MG, Hegge JO, Noble MA, Lewis DL, Herweijer H, Wolff JA. Progress toward a nonviral gene therapy protocol for the treatment of anemia. Human gene therapy. 2007;18(3):269-85.

38. Maruyama H, Ataka K, Gejyo F, Higuchi N, Ito Y, Hirahara H, et al. Long-term production of erythropoietin after electroporation-mediated transfer of plasmid DNA into the muscles of normal and uremic rats. Gene therapy. 2001;8(6):461-8.

39. Ma C, Fan Z, Gao Z, Wang S, Shan Z. Delivery of human erythropoietin gene with an adenoassociated virus vector through parotid glands to treat renal anaemia in a swine model. Gene therapy. 2017;24(11):692-8.

40. Tao Y, Zhu Q, Wang L, Zha X, Teng D, Xu L. Adeno-associated virus (AAV)-mediated neuroprotective effects on the degenerative retina: the therapeutic potential of erythropoietin. Fundam Clin Pharmacol. 2020;34(1):131-47.

41. Osada S, Ebihara I, Setoguchi Y, Takahashi H, Tomino Y, Koide H. Gene therapy for renal anemia in mice with polycystic kidney using an adenovirus vector encoding the human erythropoietin gene. Kidney international. 1999;55(4):1234-40.

42. Gao G, Lebherz C, Weiner DJ, Grant R, Calcedo R, McCullough B, et al. Erythropoietin gene therapy leads to autoimmune anemia in macaques. Blood. 2004;103(9):3300-2.

43. Roth J, Curiel D, Pereboeva L. Cell vehicle targeting strategies. Gene therapy. 2008;15(10):716-29.

44. Maumus M, Guérit D, Toupet K, Jorgensen C, Noël D. Mesenchymal stem cell-based therapies in regenerative medicine: applications in rheumatology. Stem Cell Res Ther. 2011;2(2):14.

45. Sage EK, Thakrar RM, Janes SM. Genetically modified mesenchymal stromal cells in cancer therapy. Cytotherapy. 2016;18(11):1435-45.

46. Gómez-Mauricio G, Moscoso I, Martín-Cancho M-F, Crisóstomo V, Prat-Vidal C, Báez-Díaz C, et al. Combined administration of mesenchymal stem cells overexpressing IGF-1 and HGF enhances neovascularization but moderately improves cardiac regeneration in a porcine model. Stem Cell Res Ther. 2016;7(1):94.

47. Scheibe F, Gladow N, Mergenthaler P, Tucker A, Meisel A, Prockop D, et al. Nonviral gene delivery of erythropoietin by mesenchymal stromal cells. Gene therapy. 2012;19(5):550-60.

48. Sweeney NP, Regan C, Liu J, Galleu A, Dazzi F, Lindemann D, et al. Rapid and efficient stable gene transfer to mesenchymal stromal cells using a modified foamy virus vector. Mol Ther. 2016;24(7):1227-36.

49. Dompe C, Janowicz K, Hutchings G, Moncrieff L, Jankowski M, Nawrocki MJ, et al. Epigenetic Research in Stem Cell Bioengineering-Anti-Cancer Therapy, Regenerative and Reconstructive Medicine in Human Clinical Trials. Cancers. 2020;12(4):1016.

50. Nurgali K, Jagoe RT, Abalo R. Adverse effects of cancer chemotherapy: Anything new to improve tolerance and reduce sequelae? Front Pharmacol. 2018;9:245. 
51. Parekkadan B, Milwid JM. Mesenchymal stem cells as therapeutics. Annu Rev Biomed Eng. 2010;12:87-117.

52. Coyne TM, Marcus AJ, Reynolds K, Black IB, Woodbury D. Disparate host response and donor survival after the transplantation of mesenchymal or neuroectodermal cells to the intact rodent brain. Transplantation. 2007;84(11):1507-16.

53. Lippin Y, Dranitzki-Elhalel M, Brill-Almon E, Mei-Zahav C, Mizrachi S, Liberman Y, et al. Human erythropoietin gene therapy for patients with chronic renal failure. Blood. 2005;106(7):2280-6.

54. Macieira-Coelho A. Slowing down of the cell cycle during fibroblast proliferation. Cellular Ageing: Springer; 2016. pp. 29-47.

55. Marino L, Castaldi MA, Rosamilio R, Ragni E, Vitolo R, Fulgione C, et al. Mesenchymal Stem Cells from the Wharton's Jelly of the Human Umbilical Cord: Biological Properties and Therapeutic Potential. International Journal of Stem Cells. 2019;12(2):218.

56. Bougioukli S, Saitta B, Sugiyama O, Tang AH, Elphingstone J, Evseenko D, et al. Lentiviral Gene Therapy for Bone Repair Using Human Umbilical Cord Blood-Derived Mesenchymal Stem Cells. Human gene therapy. 2019;30(7):906-17.

57. Wu X, Jiang J, Gu Z, Zhang J, Chen Y, Liu X. Mesenchymal stromal cell therapies: immunomodulatory properties and clinical progress. Stem Cell Res Ther. 2020;11(1):1-16.

\section{Figures}
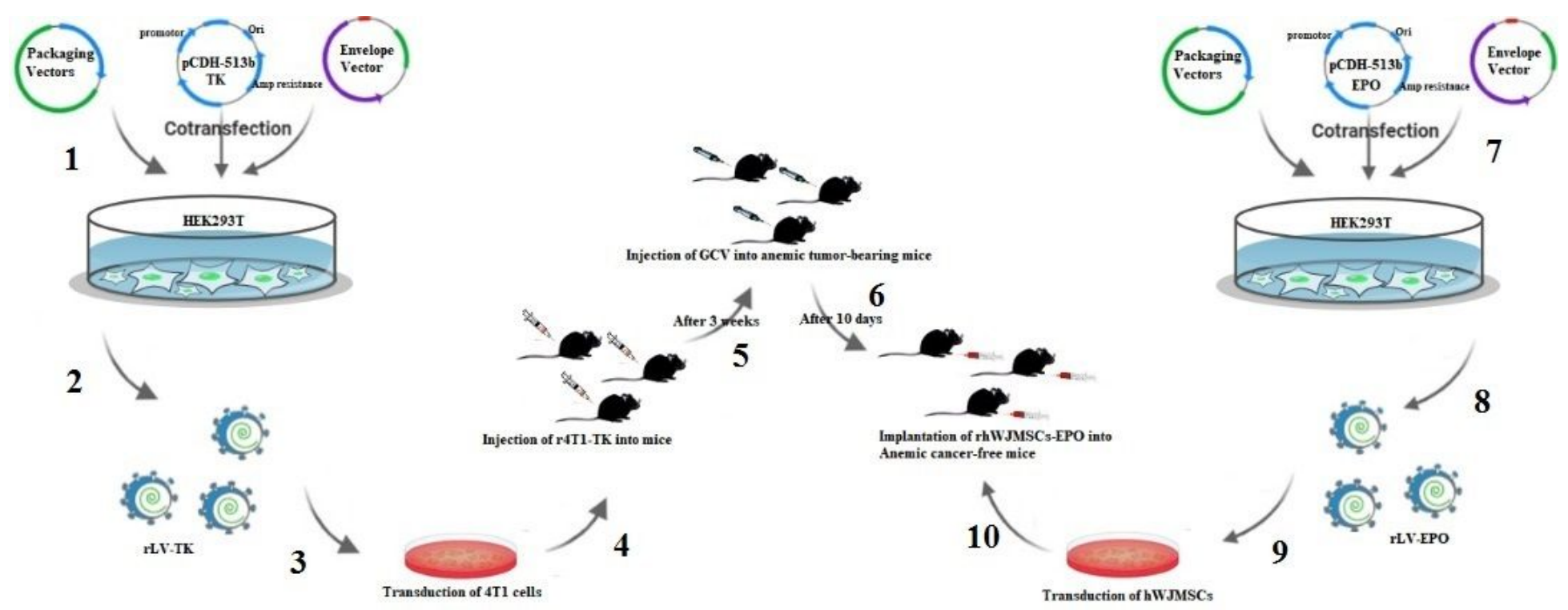

\section{Figure 1}

Schematic Presentation Constructs and Restriction Enzyme Digestion Map. In this construct, a EPO and b HSV-TK(B) mRNA were transcripts from CMV promoter,and cGFP and Puromycin mRNA were transcript from EF1a promoter. C The EPO was digested with Xbal-Apal that produced two bands of 1900 and 7800 
bp. d The HSV1-TK was digested with Spel-EcoRI that produced two bands of 1100 and $8200 \mathrm{bp}$. Note: Lane 1: Plasmid; Lane 2: Digested Plasmid; Lane M: 1 kb DNA Marker.

A

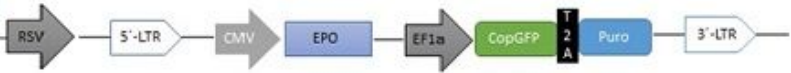

B

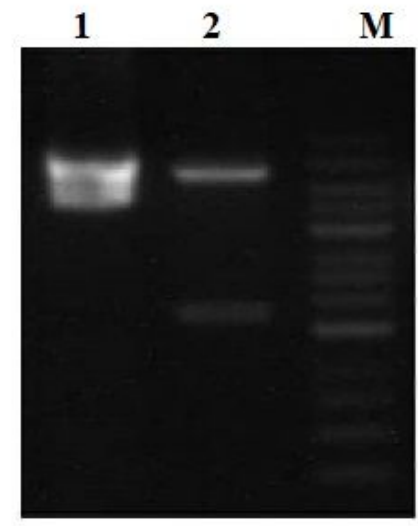

\section{M}

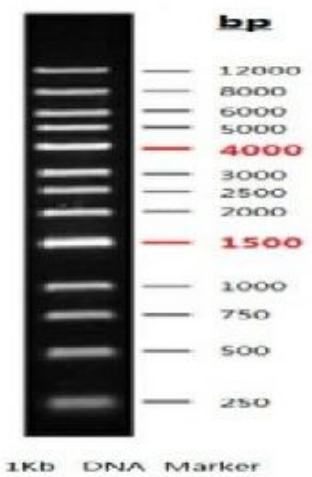

C

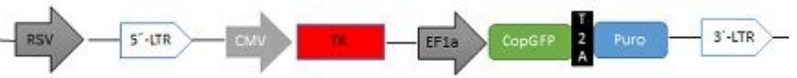

D

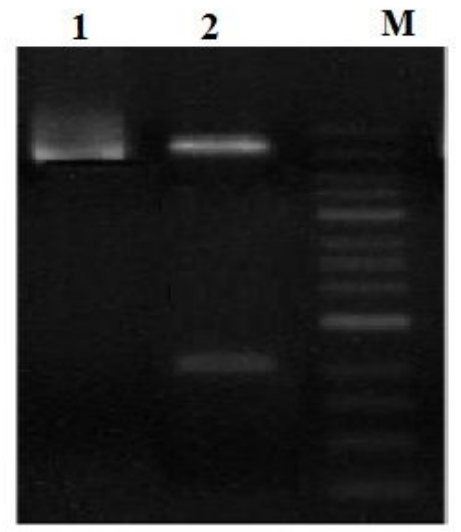

Figure 2

Isolation expansion and characterization of human Wharton's jelly mesenchymal stem cells (hWJMSCs) from umbilical cords. a Flow cytometry analysis of hWJMSCs populations showed a positive marker for CD105 and CD90 and negative marker for CD45 and CD34. Results showed that more than 95\% of hWJMSCs are positive for mesenchymal stem cell markers and negative for hematopoietic stem cell markers. b hWJMSCs were cultured in DMEM-F12 and showed fibroblasts morphology, stained mineral calcium indicated osteogenic differentiation, and oil droplets confirmed adipogenic differentiation.

A

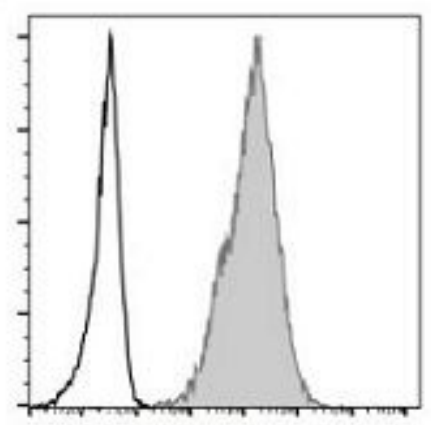

CD 105

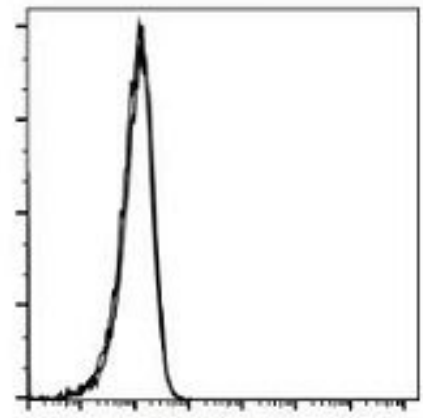

CD 34

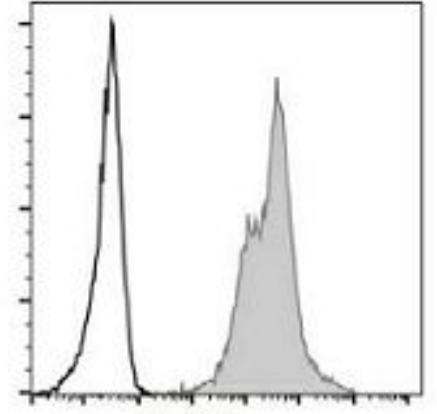

CD 90

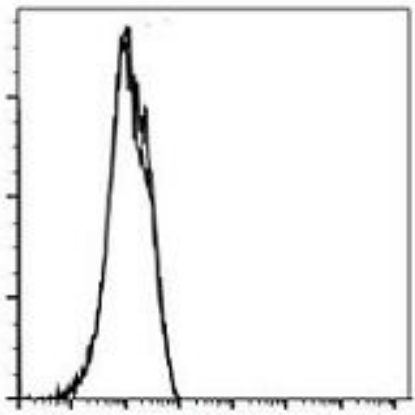

CD 45
B

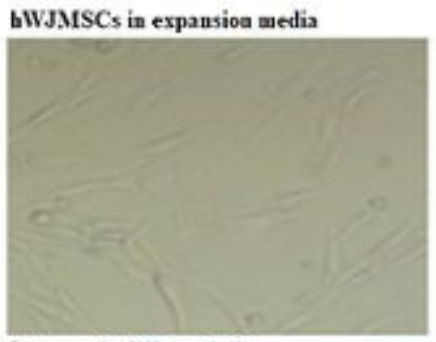

Osteogeaic differeatiation

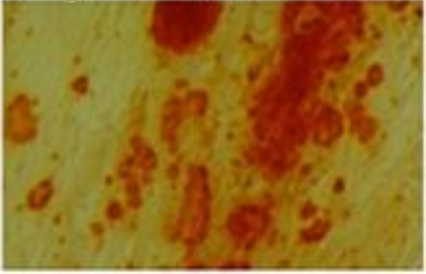

Adipogenic differentiation

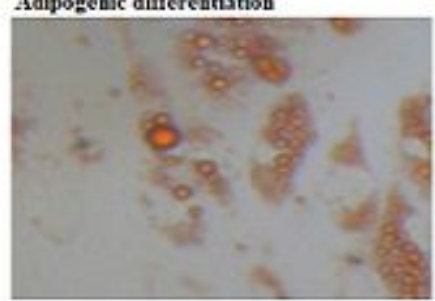




\section{Figure 3}

Transfection of lentiviral transfer vector in HEK-293T and transduction of hWJMSCs and 4T1 cells, evaluate viability with MTT test and gene expression by western blot analysis. a HEK-293 T cells were transfected with transfer vectors which showed high transfection efficacy. b hWJMSCs and 4T1 cells were transduced with recombinant lentiviral vectors,and the expression rate of GFP was visualized by fluorescent microscope. c MTT test showed in the picture, cell growth and viability are the same in transduced hWJMSCs and normal hWJMSCs cells $\left({ }^{\star} \mathrm{p}<0.05\right)$. Transduced hWJMSCs viability in different MOls has no significant versions $\left({ }^{*} \mathrm{p}<0.05\right)$. Also, cell growth and viability are the same in transduced and non-transduced $4 T 1$ cells ( ${ }^{\star} \mathrm{p}<0.05$ ). adding GCV to r4T1-TK cells cause a significant decrease in cell growth and viability. $\mathrm{d}$ Western blot images here show from transfer vector and control vector after transduction hWJMSCs. As in the first column was showed EPO and 4T1 expressed from the transfer vector. In the right column, $\beta$-actin is only detected as control.

A
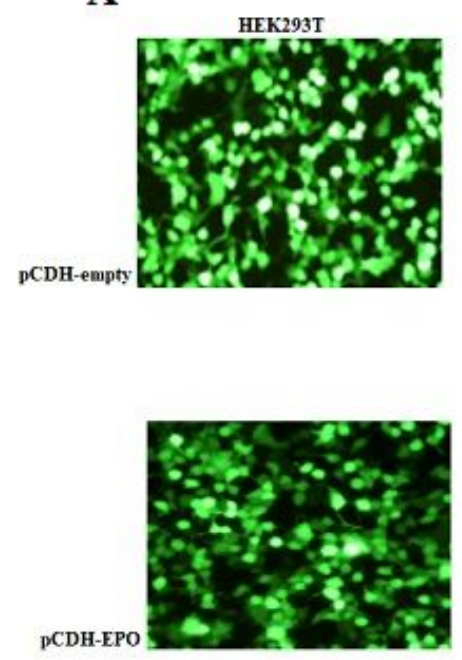

pCDH-TK

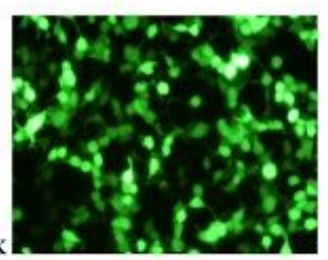

B
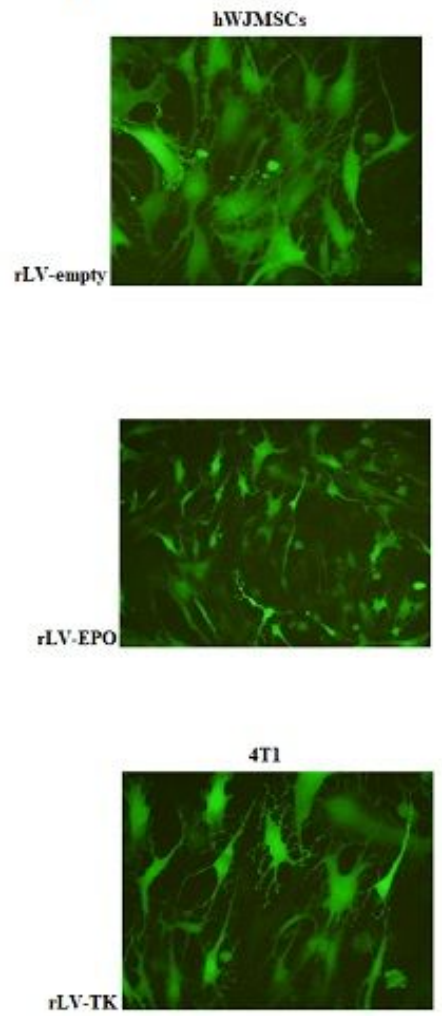

C

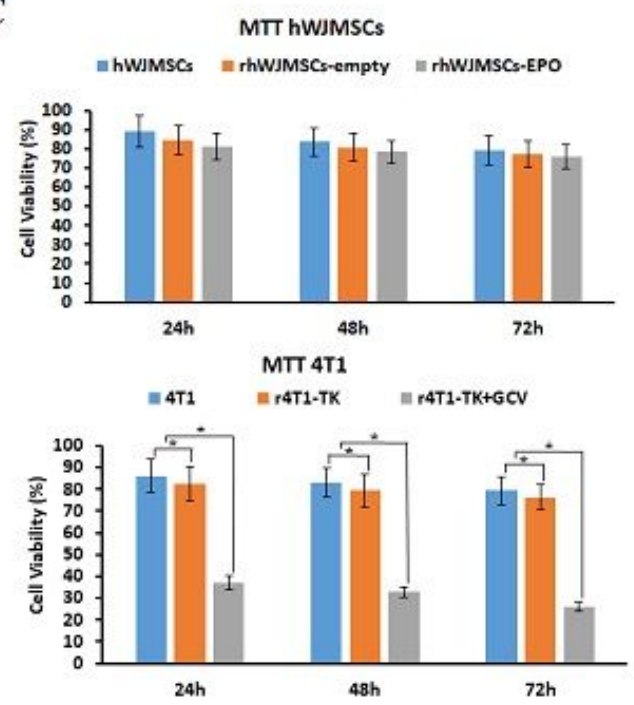

D

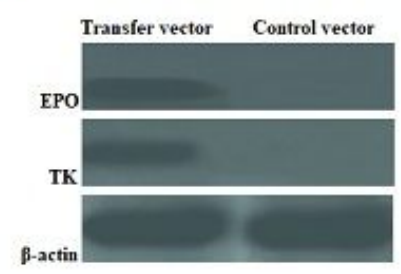

Figure 4

Cancer-related anemia (CRA) in mice following injection of r4T1-TK and Effect of GCV on tumor regression. a r4T1-TK-bearing mice showed an anemic feature with lower RBC count, $\mathrm{Hb}$, and Hct levels, and increased reticulocytosis, compared to control mice ( ${ }^{\star} p<0.05$ ). b Three tumor-bearing mice groups were treated by 10 days of GCV, and the other three groups received saline. Tumor volumes were measured two times a week until animals were sacrificed. Significant tumor regression was observed in three groups that received GCV. 
A
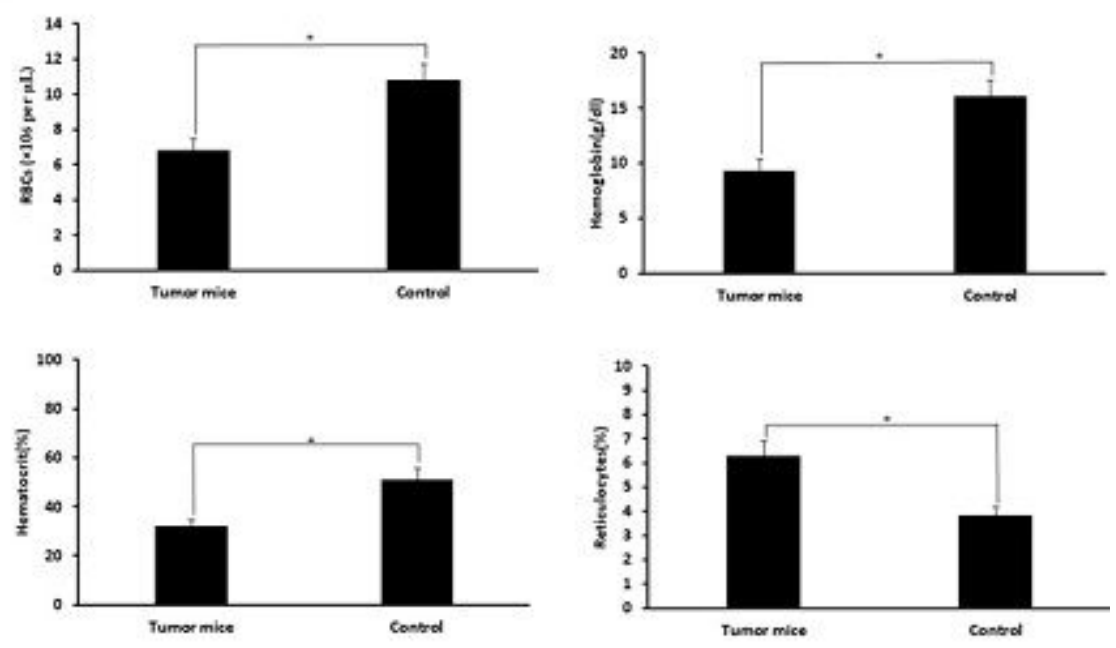

B

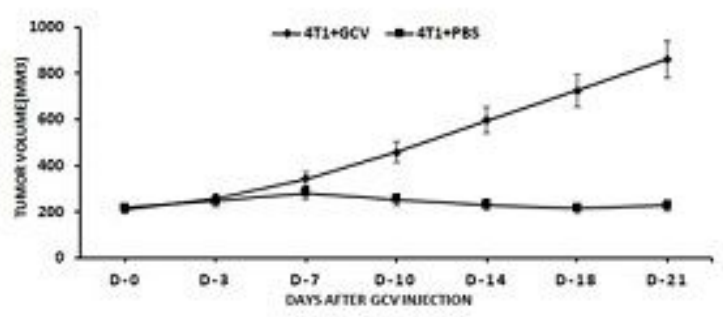

Figure 5

Time-course of serum EPO and levels of hemoglobin and hematocrit. a Studying the time course of circulating EPO after implanting 7×106 rhWJMSCs into skeletal muscle of mice. Groups A and D received rhWJMSCs-EPO; groups $B$ and $E$ received rhWJMSCs, and groups $C$ and $F$ received PBS. Both treatment groups ( $A$ and $D$ ) showed a significant increase in plasma EPO levels compared to control groups. $b$ Both treatment groups $A$ and $D$ reached a significant difference in hemoglobin levels compared to control groups. c A similar pattern of significant difference was observed in the Hct level in which groups $A$ and $D$ showed a higher Hct number compared to control groups. 

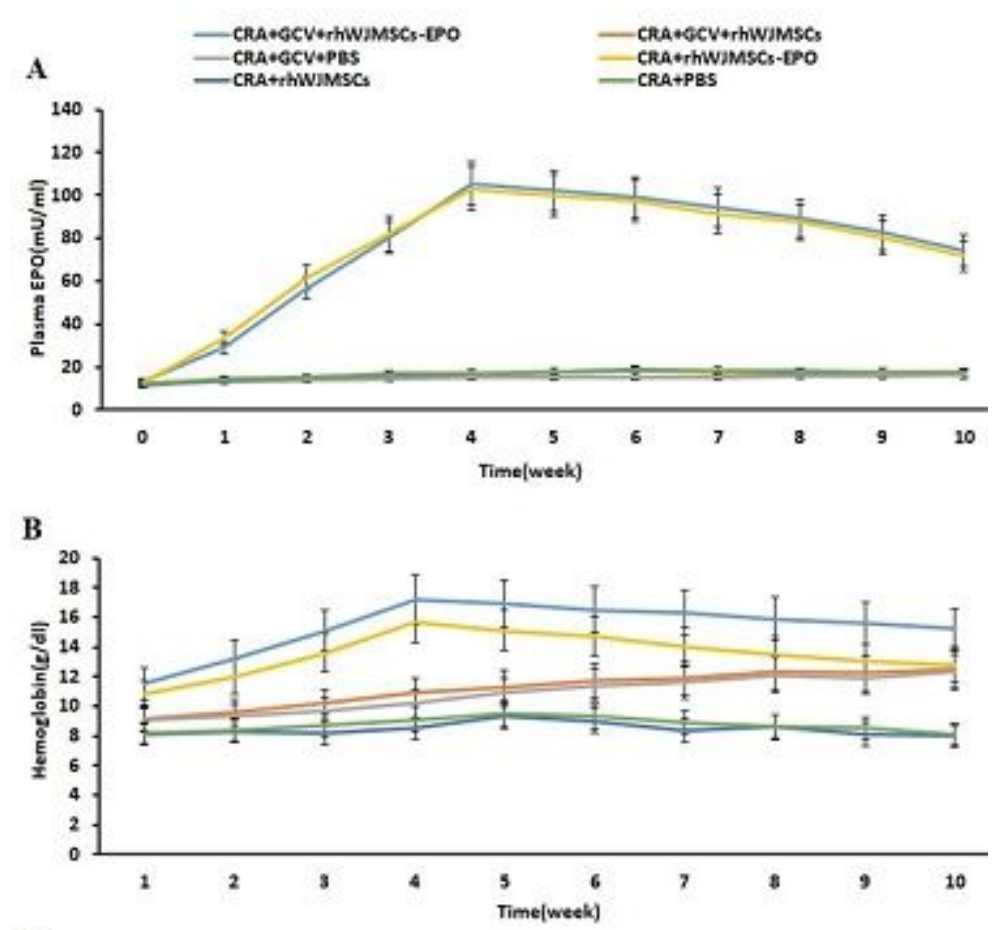

C

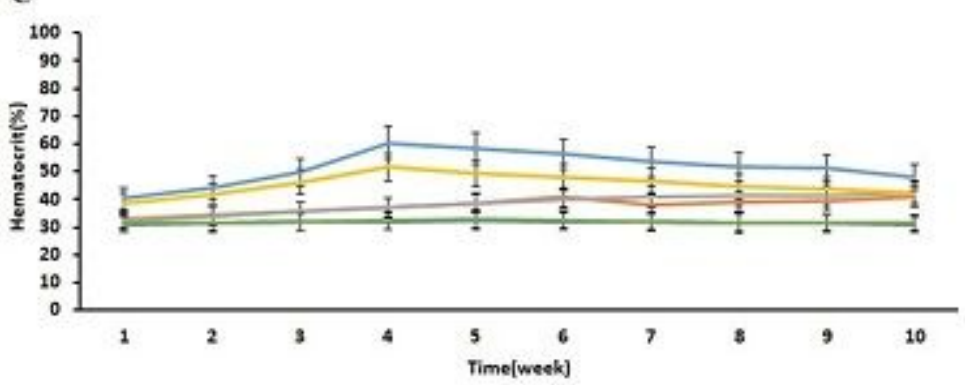

Figure 6

Time-course of serum EPO and levels of hemoglobin and hematocrit. 